\title{
Addressing Coverage Problem in Wireless Sensor Networks Based on Evolutionary Algorithms
}

\author{
Sahar Chehrazad $^{1}$, Hadi S. Aghdasi ${ }^{1}$, Negin Shariati ${ }^{2}$ and Mehran Abolhasan ${ }^{2}$ \\ ${ }^{1}$ Faculty of Electrical and Computer Engineering, University of Tabriz, Tabriz, Iran \\ ${ }^{2}$ School of Computing and Communications, UTS, Sydney, Australia
}

\begin{abstract}
Wireless Sensor Networks (WSNs) are the key part of Internet of Things, as they provide the physical interface between onfield information and backbone analytic engines. An important role of WSNs -when collecting vital information- is to provide a consistent and reliable coverage. To Achieve this, WSNs must implement a highly reliable and efficient coverage recovery algorithm. In this paper, we take a fresh new approach to coverage recovery based on evolutionary algorithms. We propose EMACB-SA, which introduces a new evolutionary algorithm that selects coverage sets using a fitness function that balances energy efficiency and redundancy. The proposed algorithm improves network's coverage and lifetime in areas with heterogeneous event rate in comparison to previous works and hence, it is suitable for using in disaster management.
\end{abstract}

Index Terms-Wireless sensor networks, internet of things, coverage recovery, evolutionary algorithms, disaster management

\section{INTRODUCTION}

Wireless sensor networks (WSNs) consist of small devices called sensor nodes which have limited sensing, processing and communicating abilities [1], [2]. Monitoring the desired area (area coverage) with the use of WSNs (as represented in Fig.1) plays a very important role in disaster management by finding the exact locations of trapped people and helps rescue teams in the optimal management of disasters. Sensor nodes gather environmental information (e.g. temperature, sound, and pictures) with the aim of fast detection of disaster, identifying and helping trapped people and helping rescue teams to provide a better management of resources [3]-[8]. Scheduling sensor nodes (selecting a subset of sensor nodes for covering whole area) in area coverage can provide us with monitoring the area for a longer time and thus more time for rescuing trapped people. Using evolutionary algorithms for scheduling sensor nodes is beneficial as they scale well to higher dimensional problems in one hand and can easily be adjusted to the problem on the other hand. Therefore these algorithms are obtained in this paper for solving the coverage problem.

Column generation method [9] uses heuristic algorithms to solve Pricing Sub problem (PS). Provided that, heuristics are unable to find a beneficial solution, integer linear programming (ILP) will be utilized to solve PS . Maximum lifetime scheduling method [10] at first, provides a multinomial time estimation procedure for the situation where the density of target points is restricted, and then a multinomial time constant factor estimation algorithm for the general case. Energy-Efficient Probabilistic Area Coverage method [11] first surveys the sensing probabilities of two points with a distance of $d$ and gets the basic mathematical relation between them. If the sensing probability of one point is greater than the specified amount, the other point is covered as well. Hybrid Genetic Algorithm Using a Forward Encoding Scheme [12] merges a genetic algorithm with schedule transition operations, termed STHGA. The greatest gene value of each chromosome is continuously grown with the quality of the solution, which pertains to the number of disjoint complete coverage sets. Hybrid Memetic Framework [13] deals with both disjoint set covers (DSC) and dynamic-coverage-maintenance (DCM) problems simultaneously

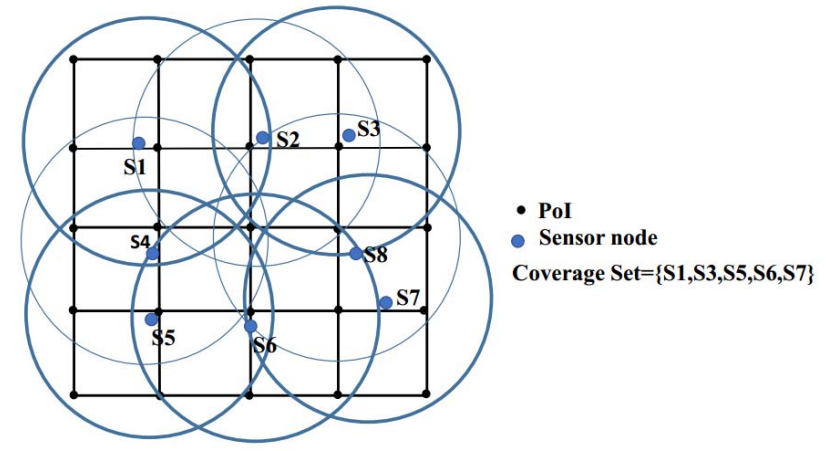

Fig. 1. Area Coverage with Wireless Sensor Networks

to maximize the lifetime of WSNs. Ant-Colony-Based Scheduling Algorithm [14] is concerned with finding the solution for the EEC (Energy-Efficient Coverage) problem with a scheduling method based on ant colony optimization in an unstructured WSN. The pheromone field is initialized with the location of sensor nodes and PoIs and the number of PoIs that can be covered by each sensor node. By doing this, the possibility of selecting sensor nodes which cover more PoIs at the beginning can be increased. In the construction graph, ant $k$ adds sensor nodes one at a time till selected sensor set covers all PoIs. At the beginning, the ACB-SA algorithm organizes the coverage set with few sensor nodes and uses them continuously for several time steps. Once the energy of the previously organized subset was nearly depleted, the ACB-SA algorithm puts some new sensor nodes in the coverage set to cover all PoIs.

All above-mentioned algorithms did not have a special look at heterogeneous event rate in their works. Since the events are distributed unequally in the area with heterogeneous event rate, if sensor nodes which are placed in the areas with more event rates are used for successive time steps, they will be depleted after a short time. Therefore, in some applications like disaster management, the network may be unable to cover some areas after a while. The proposed algorithm is based on evolutionary algorithms. It not only meets the criteria in the previous works, but also considers event rate heterogeneous in the environment. The EMACB-SA algorithm improves network's coverage and lifetime in comparison to previous ACB-SA algorithm. The organization of this paper is as follows: In Section II, System Model and a list of abbreviations used in this paper are demonstrated. In Section III theoretical analysis and detailed description of the proposed algorithm are represented. Section IV provides an overview of Simulation Results which shows that the proposed algorithm is more efficient in comparison to the existing algorithms. Finally Section V is allocated to the conclusion of the proposed EMACBSA algorithm. Table I represents a list of abbreviations used in this paper. 


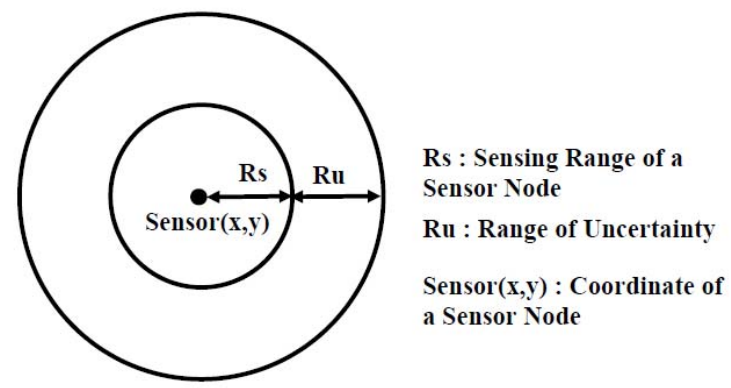

Fig. 2. Rs and Ru of a sensor node

\section{SYSTEM MODEL}

Our area of interest contains $N$ sensor nodes which are small and capable of communicating in short distances. In this paper, we used a probabilistic sensor detection model which is more realistic [14]. Sensor nodes detect the event at the Point of Interest (PoI) by the intensity of the received signal or energy. This intensity is reduced as the distance between the PoI and the sensor node increases. The probability of event detection is a decreasing function of the distance between the PoI and the center of the sensor node. The equation for this model is as follows. $\lambda i(j)$ is the detection probability of the sensor node $i$ about events at PoIj.

$$
\lambda i(j)= \begin{cases}0, & \text { if } d_{i j}>R s+R u \\ e^{-a\left(d_{i j}-R s\right)^{m}}, & \text { if } R s<d_{i j} \leq R s+R u \\ 1, & \text { if } d_{i j} \leq R s\end{cases}
$$

Where $d_{i j}$ is the Euclidean distance between the sensor node $\mathrm{i}$ and PoIj. The variables $a$ and $m$ are decay factors. $R s$ and $R u$ are sensing range of a sensor node and its range of uncertainty in event detection respectively (Fig.2). Events at PoIj are definitely detected by sensor node $I$ when $d_{i j}$ is less than $R s$. As $d_{i j}$ increases, the detection probability is reduced. When $d_{i j}$ is greater than $R u$, the detection probability becomes zero. The variables $a, m, R s$, and $R u$ depend on the characteristics of the sensor node and the environment.

\section{PROPOSED ALGORITHM}

In this paper, the proposed algorithm improves area coverage and extends network's lifetime. In the EMACB-SA algorithm, the selection of new coverage set occurs in each time step. A new fitness function is presented to balance energy efficiency and redundancy. A coverage set in which sensor nodes have more remaining energy and cover less redundant PoIs is selected in each time step. The importance of these criterion can be defined by coefficients $a$ and $b$ as represented in the equation 3 . These coefficients get the values between zero and 1. If the remaining energy is more important to us, a higher amount is given to a. Else, $b$ will get a higher amount. In order to further reduce the redundancy, a greedy algorithm is used in the EMACB-SA algorithm due to which each ant adds selected sensor node to its coverage set if it covers any new PoI.

\section{A. Efficient Modified Ant Colony Based Scheduling Algorithm (EMACB-SA)}

Suppose that a lot of sensor nodes are distributed in the environment. Table II represents the list of acronyms which are used in this paper.

At the beginning to initialize the pheromone field, the location of sensor nodes and PoIs are calculated [14] and put in two matrix named $L S e n$ and LPoI respectively. At the beginning of each time
TABLE I

List OF ABBREVIATIONS

\begin{tabular}{|c|c|}
\hline Abbreviation & Definition \\
\hline ACB-SA & Ant Colony Based Scheduling Algorithm \\
\hline DCM & Dynamic-Coverage-Maintenance \\
\hline DSC & Disjoint Set Covers \\
\hline $\mathrm{EC}$ & $\begin{array}{l}\text { Energy for Coverage in each active time step } \\
\text { which equals to } 0.1\end{array}$ \\
\hline EEC & Energy-Efficient Coverage \\
\hline $\mathrm{EI}(\mathrm{ts})$ & $\begin{array}{l}\text { Initial Energy which is equal to } 1 \text { at } t s=0 \\
\text { and the remaining energy of sensor }\end{array}$ \\
\hline EMACB-SA & $\begin{array}{l}\text { I at each time step (ts) is saved in it. } \\
\text { Efficient Modified Ant Colony Based } \\
\text { Scheduling Algorithm }\end{array}$ \\
\hline ILP & Integer Linear Programming \\
\hline LPoI & Location of PoI \\
\hline LSen & Location of Sensor \\
\hline MaxIter & Maximum Iteration \\
\hline NA & Number of Ants \\
\hline NP & Number of PoIs \\
\hline NS & Number of Sensor nodes \\
\hline PoI & Point of Interest \\
\hline PS & Pricing Sub problem \\
\hline Rs & Sensing Range of sensor node \\
\hline $\mathrm{Ru}$ & Range of Uncertainty \\
\hline ts & time step \\
\hline WSNs & Wireless Sensor Networks \\
\hline
\end{tabular}

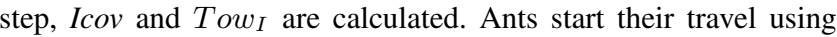
the proposed construction graph. Ant $k$ chooses a sensor node by roulette-wheel selection randomly in which each sensor node $I$ has the selection probability of $\mathrm{P}(\mathrm{I})$ :

$$
P(I)=\text { Tow }_{I} / \sum_{n=1}^{N S} \operatorname{Tow}_{I}
$$

Each ant selects sensor nodes one at a time. Then greedy algorithm checks whether the selected sensor node covers any new PoI. At the beginning of the EMACB-SA algorithm, the PoIs covered by each sensor node are saved in a matrix named Sen-POI. Total number of PoIs covered by selected sensor nodes are kept in another matrix named Ant-POI. Each time a new sensor node is selected and added to the coverage set, Ant-POI is updated. Therefore uncovered PoIs can be found. The sensor node is added to the coverage set if it covers any new PoI. Otherwise, it is dropped. A simple example of the greedy algorithm is demonstrated in Fig.3. This will prevent the selection of sensor nodes which have no benefit. By using this method, sensor nodes are not activated uselessly and the lifetime of the network is increased.

If ant $k$ finds a set of sensor nodes which cover all PoIs, ant $k+1$ will start its travel. After all ants (MaxAnt) finish their selections, the best solution can be found with the use of proposed fitness function which balances energy efficiency and redundancy. In order to find the best solution two criterion, the cost of the selected sensor nodes and redundancy, should be considered. The higher the remaining energy of the sensor node $I$ is, the lower 
TABLE II

LIST OF ACRONYMS

\begin{tabular}{|c|c|}
\hline Acronyms & Definition \\
\hline $\mathrm{a}, \mathrm{b}$ & Numbers between zero and one and $a+b=1$ \\
\hline Bcost & $\begin{array}{l}\text { Matrix which contains the lowest } C_{A}(\mathrm{ka}) \\
\text { in each iteration }\end{array}$ \\
\hline Bcover & $\begin{array}{l}\text { Matrix which contains the coverage set with } \\
\text { lowest } C_{A}(\mathrm{ka}) \text { in each iteration }\end{array}$ \\
\hline$C_{A}(\mathrm{ka})$ & Cost of selected sensor set by ant ka \\
\hline $\operatorname{Cost}_{W}$ & $\begin{array}{l}\text { Worst cost which happens when the ant selects all } \\
\text { sensors as the solution of the problem }\end{array}$ \\
\hline $\operatorname{Icov}(\mathrm{ts})$ & $\begin{array}{l}\text { A matrix which includes the number of POIs that is } \\
\text { covered by sensor I and is calculated at } \\
\text { the beginning of each time step }\end{array}$ \\
\hline $\operatorname{Cost}(\mathrm{I})$ & Cost of sensor node I that is equal to $K^{E I}$ \\
\hline K & random number between zero and one \\
\hline $\mathrm{n}$ & $\begin{array}{l}\text { Number of sensor nodes in the selected coverage set } \\
\text { by ant }\end{array}$ \\
\hline $\operatorname{Red}_{A}(\mathrm{ka}, \mathrm{ts})$ & $\begin{array}{l}\text { Number of times that each PoI is covered by more } \\
\text { than one sensor node in time step (ts) which } \\
\text { equals to } \sum_{n=1}^{n} I \operatorname{cov}(t s)-N P\end{array}$ \\
\hline $\operatorname{Red}_{W}$ & $\begin{array}{l}\text { Worst redundancy happens when the ant selects all } \\
\text { sensor nodes as the solution of the problem which } \\
\text { equals to } \sum_{n=1}^{N S} I \operatorname{cov}(1)-N P\end{array}$ \\
\hline $\operatorname{Tow}_{I}(\mathrm{ts})$ & $\begin{array}{l}\text { Pheromone field which is updated for each sensor } \\
\text { node } \mathrm{I} \text { at the beginning of each time step which } \\
\text { equals to } E I(t s) \times I \operatorname{cov}(t s)\end{array}$ \\
\hline
\end{tabular}

$\operatorname{Cost}(I)$ will be. In this case the $C_{A}(\mathrm{ka})$ will get a lower value. This increases the probability of selecting the coverage set in which sensor nodes have higher remaining energy. On the other hand, as the redundancy of the selected coverage set increases, the $C_{A}(\mathrm{ka})$ gets a higher value. This reduces the probability of coverage set with higher redundancy.Following fitness function by adaptive weighted sum method [15] was proposed to make a tradeoff between these criterion:

$$
\begin{aligned}
C_{A}(k a)= & a \times\left(\left(\sum_{I=1}^{n} \operatorname{Cost}(I)\right) / \operatorname{Cost}_{W}\right) \\
& +\left(b \times \operatorname{Red}_{A}(k a, t s)\right) / \operatorname{Red}_{W}
\end{aligned}
$$

The selected coverage set with the lowest $C_{A}(\mathrm{ka})$ and its cost are saved in two matrix named Bcover (Best cover) and Bcost (Best cost) respectively. In the next iteration, Tow $_{I}$ is updated for the sensor nodes in Bcover as follows:

$$
\operatorname{Tow}_{I}=\text { Tow }_{I}+\left(B \cos t / \sum_{k a=1}^{N A} C_{A}(k a)\right)
$$

In the next iteration, all above-mentioned works are done. The ant with the lowest $C_{A}(\mathrm{ka})$ is selected. If its cost is lower than the one saved in Bcost, it will be replaced by Bcost. Then the selected coverage set in new iteration will be replaced by the one in Bcover. For selecting a coverage set in each time step, MaxIter iteration is completed and the coverage set which is in the matrix Bcover is activated in that time step. At the end of each time step, EC (a predefined amount) is reduced from the remaining energy $(E I)$ of each active sensor node. if there is a sensor node with $E I<E C$, the percent of lost coverage with the death of that sensor node is calculated (the percent of PoIs that are not covered anymore is

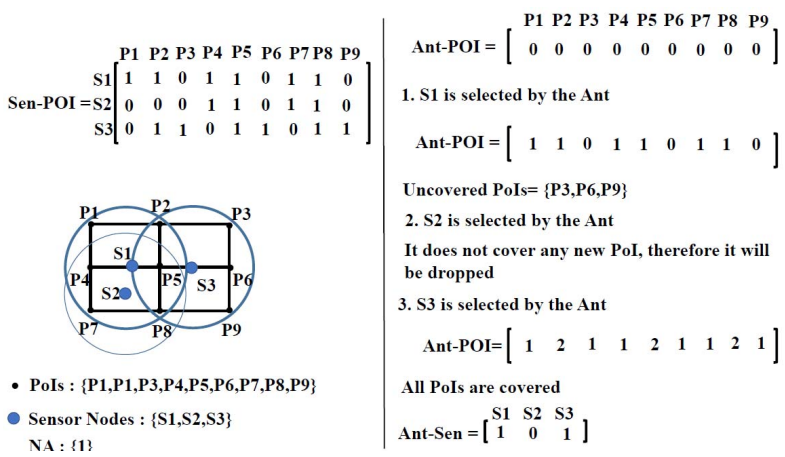

Fig. 3. An Example of the Greedy Algorithm

calculated and reduced from the coverage of the last time step). Finally, dead sensor nodes and uncovered PoIs are omitted from the matrix of sensor nodes and PoIs respectively. Otherwise, it is continued in the next time step. It is also checked at the end of each time step, whether all sensor nodes are dead. In this case, the network is dead and our algorithm stops working. Otherwise, it is continued in the next time step.

In the proposed algorithm, the death time of sensor nodes is delayed, and sensor nodes which cover more PoIs can be used in several time steps. In fact, it enables us to have full coverage of the environment in WSNs for a longer time. The flowchart of the EMACB-SA algorithm is represented in Fig.4.

\section{SIMULATION RESULTS}

All simulations were implemented on a PC with an Intel(R) Core i7-5500u operating at $3 \mathrm{GHz}$ and $12 \mathrm{~GB}$ of RAM. We implemented the simulator in MATLAB. The network is modelled as a $(100 \times$ $100)$ square grid in a $(100 \mathrm{~m} \times 100 \mathrm{~m})$ Euclidean plane. 40 sensor nodes and 10000 PoIs are located on the grid points respectively (one PoI on each point). The sensing range of sensor nodes $(R s)$ and Range of uncertainty $(R u)$ are both considered $20 \mathrm{~m}$. Both $a$ and $m$ are set to 0.5 . EI is set to one at the beginning and it is saved in a matrix named $E$ with the size of $1 \times 40$. In each time step, $E C$ is reduced from $E I$ of all active sensor nodes. In the simulation, 40 ants are used. The matrix Sen-POI with the size of $40 \times 10000$ includes PoIs which are covered by each sensor node. The matrix Ant-Sen with the size of $40 \times 40$ includes sensor nodes which are selected by each ant. The matrix Ant-POI with the size of $40 \times$ 10000 includes PoIs which are covered after selection of sensor nodes. Two scenarios are considered for simulating the proposed algorithm to illustrate its efficiency in disaster management. As ant colony algorithms are based on random selection of sensor nodes, the proposed algorithm was run several times and the simulation results were saved in the structure. By averaging the saved results for both scenarios, the area coverage, the number of all sensor nodes (active \& sleep) and redundancy were computed. To evaluate the performance of the EMACB-SA algorithm, it simulation results were compared with the ACB-SA algorithm.

\section{A. First Scenario}

In this scenario, each sensor node senses one event at each active time step and $E C=0.1$ is reduced from its $E I$. Sensor nodes are distributed in the area randomly to cover 10000 PoIs. In the EMACB-SA algorithm, the new coverage set is selected in each time step. In the ACB-SA algorithm, the selected coverage set is activated for 10 successive time steps. All sensor nodes have died at the end of 10th-time step. Therefore, the new coverage set is selected in each ten-time steps. 

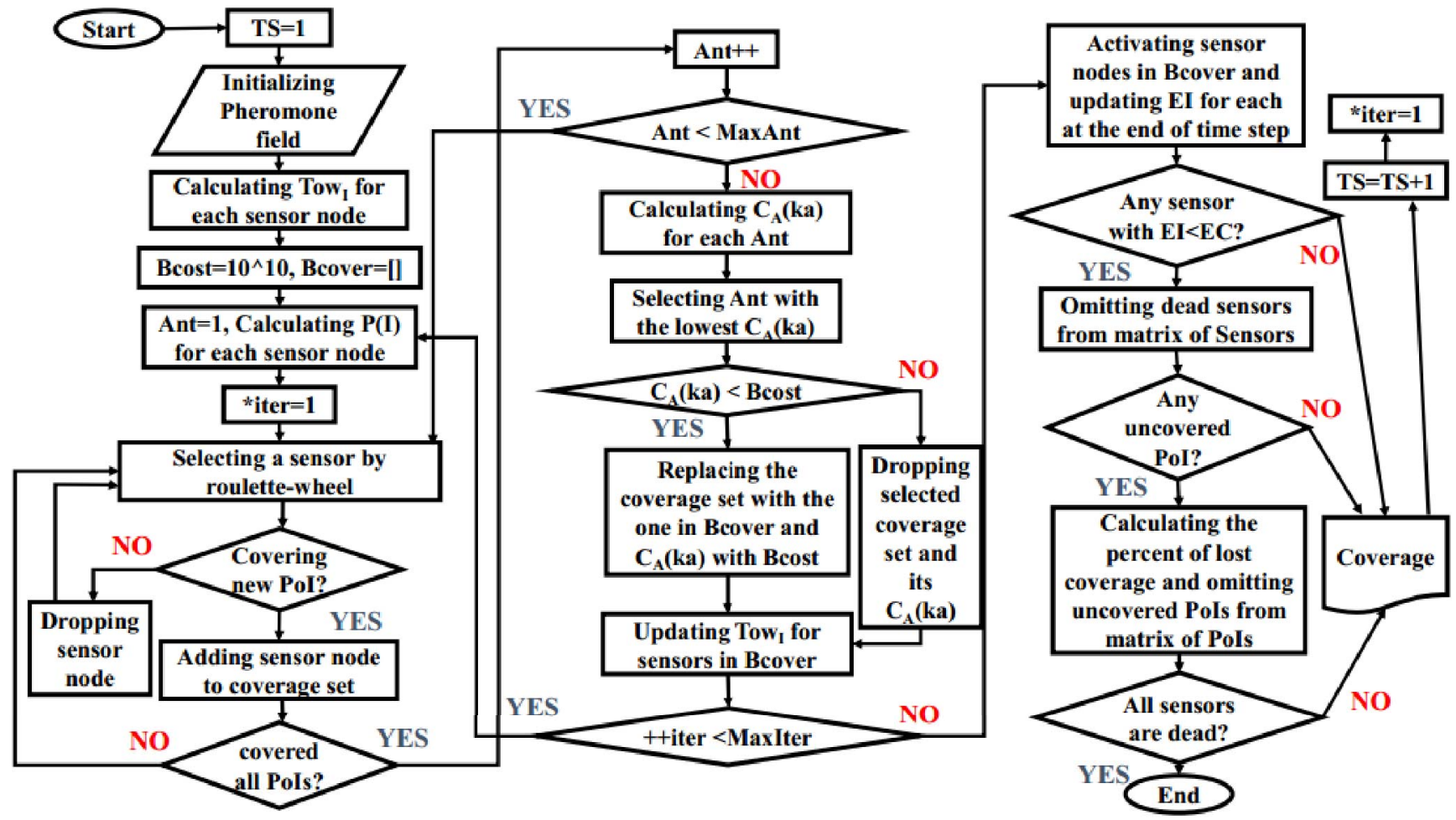

Fig. 4. Flowchart of the proposed algorithm

\section{B. Second Scenario}

In this scenario, some sensor nodes are in the regions with heterogeneous event rate. These sensor nodes sense more than one event at each active time step. Consequently, $E C=0.2$ is reduced from their $E I$. Other sensor nodes sense one event at each active time step, and $E C=0.1$ is reduced from their $E I$. Sensor nodes are distributed in the area randomly to cover 10000 PoIs. In the EMACB-SA algorithm, the new coverage set is selected in each time step. In the ACB-SA algorithm, if any sensor node in the selected coverage set belongs to a region with heterogeneous event rate, the coverage set will be activated for five successive time steps. At the end of the fifth-time step, some sensor nodes are dead. Sensor nodes which belong to the region with homogenous event rate still have $E I=0.5$ and will be activated in another time steps. If the selected coverage set includes sensor nodes from both regions and some sensor nodes have $E I=0.5$, it can be activated only for 5 successive time steps. Therefore, in the ACB$\mathrm{SA}$ algorithm, the new coverage set is selected in each ten or five-time steps.

\section{Area Coverage}

The results in Fig.5 indicate that in the first scenario, the coverage of the ACB-SA algorithm reaches to less than 50 at 50th-time step. This occurs at 63rd time step in the EMACB$\mathrm{SA}$ algorithm. It can be concluded that the EMACB-SA algorithm is 1.26 times better than the ACB-SA algorithm. In the second scenario, the coverage of the ACB-SA algorithm reaches to less than 30 at 39th-time step, while in the EMACB-SA algorithm this occurs at 50th time step. It can be concluded that EMACB-SA algorithm is 1.3 times better than the ACB-SA algorithm in this case.

\section{Number of all sensor nodes (active \& sleep)}

Fig.6 demonstrates that the number of alive sensor nodes in the EMACB-SA algorithm is 1.23 times more than the ACB-

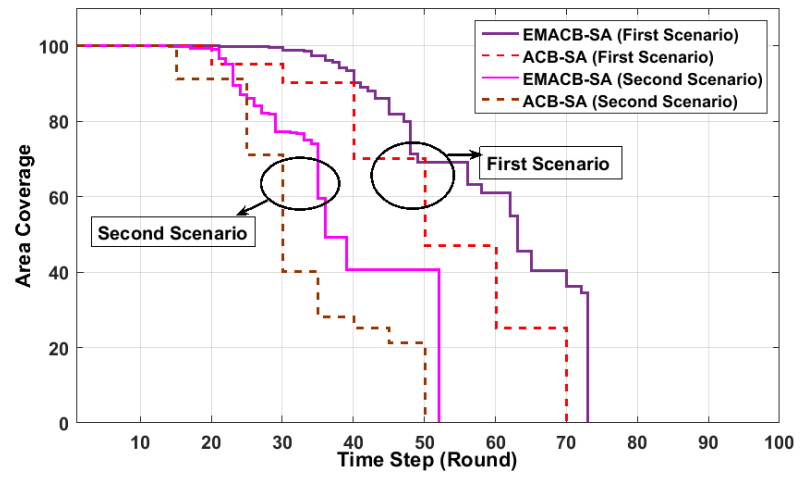

Fig. 5. Area Coverage in both scenarios

SA algorithm. The reason for the early death of sensor nodes in the ACB-SA algorithm is activating selected coverage set for successive time steps. In the EMACB-SA algorithm, the death time of first sensor node occurs at 17 th steps, while this occurs in the ACB-SA algorithm at 10th-time step. It can be deduced that the death time of first sensor node using the EMACB-SA algorithm occurs 1.7 times later than the ACB-SA algorithm.

In the second scenario, the death time of first sensor node occurs at 15th time step in the EMACB-SA algorithm, while this occurs at the 10th-time step in the ACB-SA algorithm. It is derived that the death time of first sensor node using the EMACB-SA algorithm occurs 1.5 times later than the ACB-SA algorithm.

\section{E. Redundancy}

Fig.7 (the first scenario) illustrates that the redundancy of the EMACB-SA algorithm is 7 percent better than the ACB-SA algorithm. After 50th time step, the redundancy of the EMACB-SA algorithm is 98 percent better than the ACB-SA algorithm.

In the second scenario (Fig.8), the redundancy of the EMACB$\mathrm{SA}$ algorithm is 1.73 times higher than the ACB-SA algorithm 


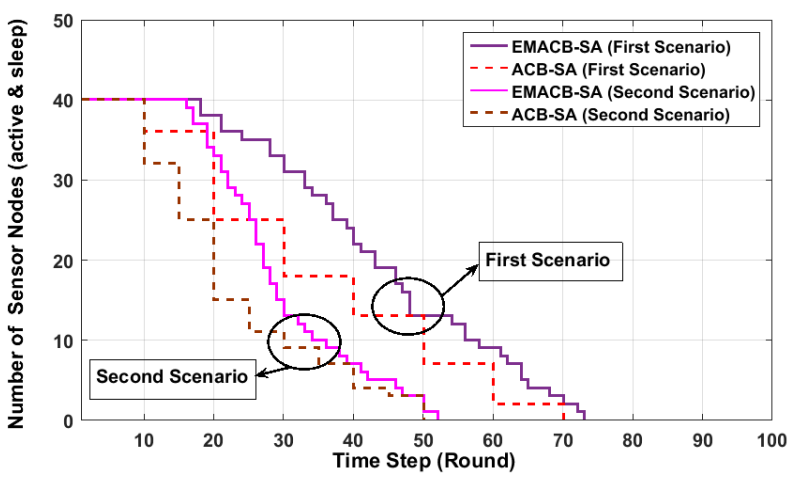

Fig. 6. Number of sensor nodes (active \& sleep) in both scenarios

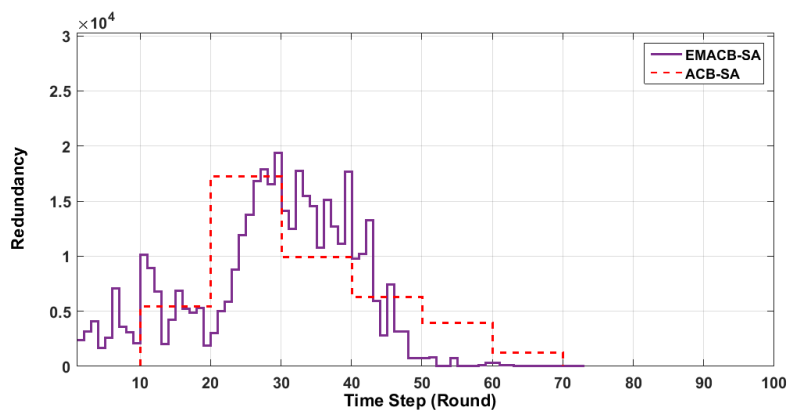

Fig. 7. Redundancy in the environment with homogeneous event rate

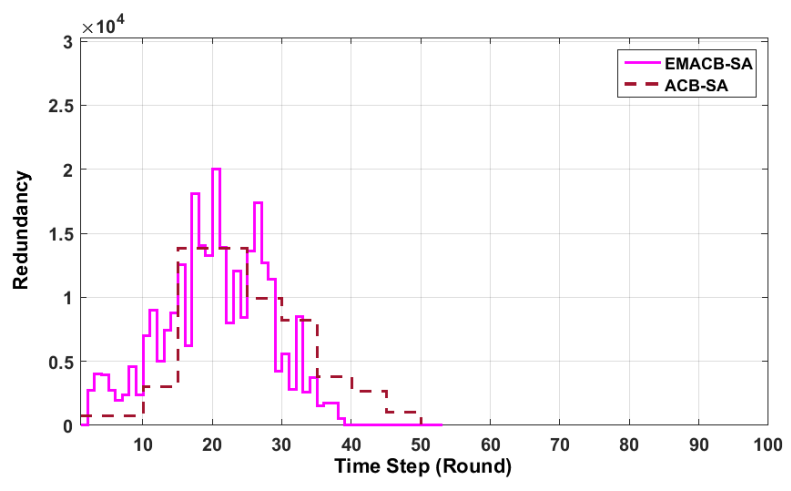

Fig. 8. Redundancy in the environment with heterogeneous event rate

till 29th-time step. After 29th time step, the redundancy of the EMACB-SA algorithm is 66 percent better than the ACB-SA algorithm.

\section{CONCLUSION}

This paper presented EMACB-SA algorithm considering different scenarios. In the first scenario, the event rate is homogenous while in the second scenario, the heterogeneous event rate is considered. For both scenarios, the area coverage, number of sensor nodes (active \& sleep) and redundancy were computed. In the proposed algorithm, a new coverage set is selected at each time step which increases the redundancy. In order to reduce the redundancy, a new fitness function and a greedy algorithm was proposed. Each sensor node is checked while being selected. The selected sensor node will be added to ant's coverage set if it covers a new PoI. Otherwise, the sensor node will be dropped.

Simulations results prove that the EMACB-SA algorithm has increased the area coverage as well as the network's lifetime. It delayed the death time of the first sensor node. Hence the proposed algorithm is a promising solution for area coverage in the environments with heterogeneous event rate.

\section{REFERENCES}

[1] I. F. Akyildiz, W. Su, Y. Sankarasubramaniam and E. Cayirci, A survey on sensor networks, IEEE Communications Magazine, Aug. 2002.

[2] I. F. Akyildiz and I. H. Kasimoglu, Wireless sensor and actor networks: Research Challenges, Broadband and Wireless Networking Laboratory, School of Electrical and Computer Engineering, Georgia Institute of Technology, 19 May 2004.

[3] P. Uplap and P. Sharma, Review of Heterogeneous/Homogeneous Wireless Sensor Networks and Intrusion Detection System Techniques, Proc. of Int. Conf. on Recent Trends in Information, Telecommunication and Computing, ITC, 2014.

[4] E. Cayirci and T. Coplu, SENDROM: sensor networks for disaster relief operations Management, Wireless Networks, pp.409-423, 2007.

[5] S. Saha and M. Matsumoto, A framework for disaster management system and protocol for rescue operation, TENCON 2007 - 2007 IEEE Region 10 Conference, 2007.

[6] G. Tuna, V.C. Gungor and K. Gulez, An autonomous wireless sensor network deployment system using mobile robots for human existence detection in case of disasters, Ad Hoc Networks, pp.54-68, 2014.

[7] M. Erd, Event monitoring in emergency scenarios using energy efficient wireless sensor nodes for the disaster information management, International Journal of Disaster Risk Reduction, pp.33-42, 2016.

[8] E. Ergen, Blockage assessment of buildings during emergency using multiple types of sensors, Automation in Construction, pp.71-82, 2015.

[9] F. Castano, A. Rossi, M. Sevaux and N. Velasco, A column generation approach to extend lifetime in wireless sensor networks with coverage and connectivity constraints, Elsevier Computers and Operation research, pp.1-11, 2013.

[10] Z. Lu, W. Li and M. Pan, Maximum lifetime scheduling for target coverage and data collection in wireless sensor networks, IEEE Transactions on Vehicular Technology, pp.1-14, 2014.

[11] Q. Yang, J. Li, J. Chen and Y. Sun, Energy-Efficient Probabilistic Area Coverage In Wireless Sensor Networks, IEEE Transactions on Vehicular Technology, pp.1-11, 2014.

[12] X. Hu, J. Zhang and Y. Yu, Hybrid Genetic Algorithm Using a Forward Encoding Scheme for Lifetime Maximization of Wireless Sensor Networks, IEEE Transactions On Evolutionary Computation, vol. 14 , no. 5 , pp.766-781, 2010.

[13] C. Chen, S. Chandra and C. Chuang, Hybrid Memetic Framework for Coverage Optimization in Wireless Sensor Networks, IEEE Transactions On Cybernetics, pp.1-14, 2014

[14] JW. Lee and JJ. Lee, Ant-Colony-Based Scheduling Algorithm for Energy-Efficient Coverage of WSN, IEEE Sensors Journal, vol.12, no.10, pp.3036-3046, 2014.

[15] X. Yu and M. Gen, Introduction to Evolutionary Algorithms, Springer-Verlag London Limited, 2010. 